\title{
Post-immunotherapy combined operative technique with an anterior surgical approach and robot-assisted lobectomy for an anterior superior sulcus tumor-case report
}

\author{
Marko Kostic $^{1,2}$, Matthieu Sarsam ${ }^{1,3}$, Benjamin Bottet $^{1}$, Jean-Marc Baste ${ }^{1}$ \\ ${ }^{1}$ Department of Thoracic Surgery, Rouen University Hospital, Rouen, France; ${ }^{2}$ Clinic for Thoracic Surgery, Clinical Center of Serbia, Belgrade, \\ Serbia; ${ }^{3}$ Department of Cardiac Surgery, Rouen University Hospital, Rouen, France \\ Correspondence to: Marko Kostic. Clinic for Thoracic Surgery, Clinical Center of Serbia, Koste Todorovica 26, 11000 Belgrade, Serbia. \\ Email: kostmarko@gmail.com.
}

\begin{abstract}
The mini-invasive approach to superior sulcus tumors is an uncommon procedure that is still far from standardization. We describe a sequential surgical technique as robotic-assisted thoracoscopic surgery (RATS) lobectomy combined with first achieved exploratory surgery in our case, modifying the usual procedure, providing good access to both the apical and hilar sites in a less invasive manner, that allows safer dissection and better visualization, which dramatically contribute to the safety of this complex surgery. A 58-year-old man presented with right superior sulcus tumor, suspecting of invading the subclavian artery. After induction immunotherapy he underwent two stage surgical procedures, vascular open exploration, and control of the subclavian artery, which was none invaded by tumor and then robotic assisted thoracoscopic right upper lobectomy. The final pathology was ypT1aN0 adenocarcinoma. The technical aspects and indication for two stage or sequential surgery are discussed in this article, the advantages, and limitations of this procedure. We believe that by reducing the invasiveness of surgery itself, we could offer patients in some selected cases a more advanced approach. This sequential surgery, as delayed two-step procedures could optimize the treatment, secures operation, and diminishes intraoperative and postoperative complications.
\end{abstract}

Keywords: Superior pulmonary sulcus tumor; immunotherapy; sequential surgery; robotic-assisted thoracoscopic surgery (RATS); case report

Received: 08 April 2020. Accepted: 27 July 2020; Published: 20 January 2022.

doi: 10.21037/jovs-20-95

View this article at: http://dx.doi.org/10.21037/jovs-20-95

\section{Introduction}

This report describes technical details of an emerging new surgical procedure consisting in a hybrid technique for treatment of anterior superior sulcus tumor. Surgical treatment can be difficult due to the invasion of adjacent structures, such as the brachial plexus, subclavian vessels and spine (1). Video-assisted thoracoscopic surgery (VATS) and robotic-assisted thoracoscopic surgery (RATS) has replaced thoracotomy as a standard surgical technique for lung cancers. Recently, a combined operative technique with an anterior surgical approach and VATS for anterior superior sulcus tumors was introduced (2) but here we want to introduce a new possible surgical philosophy as sequential surgery, which include exploratory neck cervicotomy for resolving possible tumor interference with vessels and then consecutive RATS upper lobectomy. We present the following case in accordance with the CARE reporting checklist (available at https://jovs.amegroups. com/article/view/10.21037/jovs-20-95/rc).

\section{Case presentation}

This is the case of a 58 -year-old male patient, with 60 pack/years smoking history who had alteration of his general condition losing $10 \mathrm{~kg}$ in 2 months without other specific conditions in his medical history, presented with a 

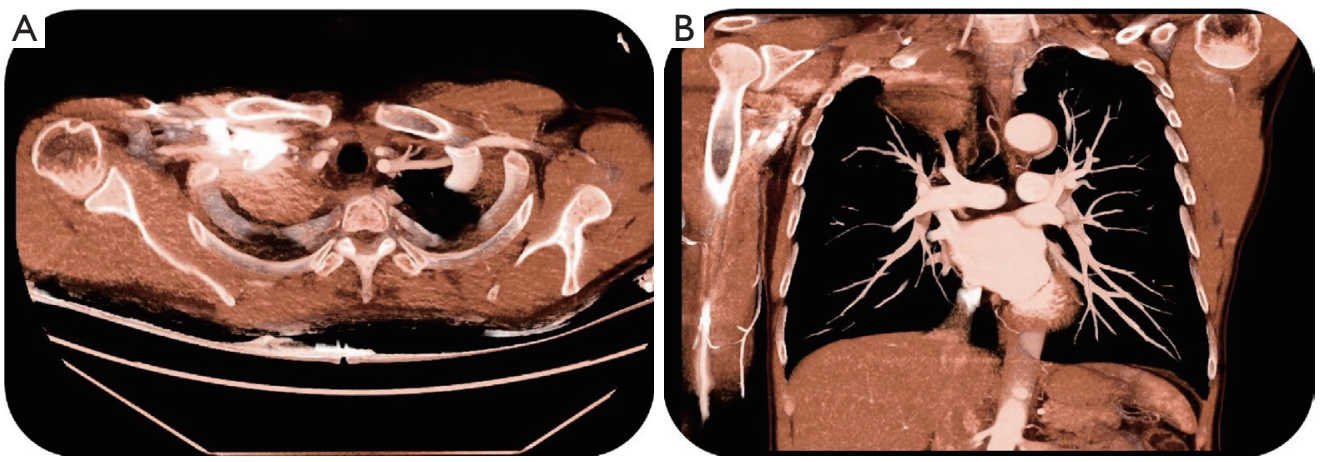

Figure 1 Preoperative computed tomography CT suggesting a superior sulcus tumor that may invade the wright subclavian artery (A) wright apical mass, and (B) infiltration of the soft tissues in the right subclavicular and supraclavicular region.

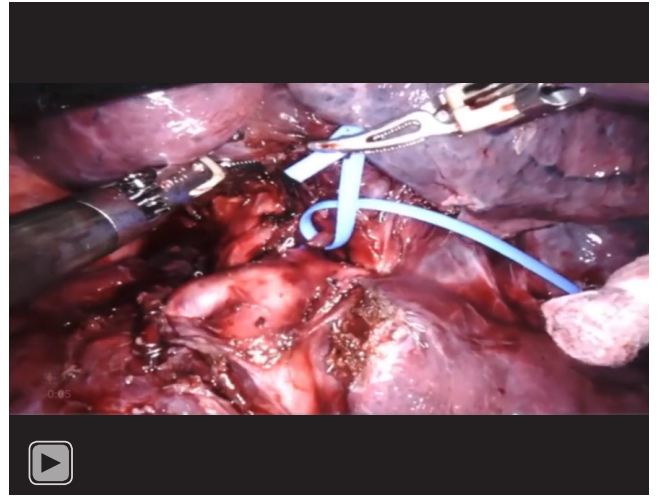

Video 1 Combined operative technique for right superior sulcus tumor.

right superior sulcus tumor, with a single metastatic lesion in the left adrenal gland. In September 2017, biopsy of the pulmonary mass revealed lung adenocarcinoma.

The patient was initially treated with $\mathrm{L} 1$ pembrolizumab as first-line therapy for stage IV disease. High-speed adrenal insufficiency under immunotherapy has occurred 6 month later in March 2018 and was treated by fludrocortisone $(50 \mu \mathrm{g} \times 1)$ and hydrocortisone $(20 \mathrm{mg} \times 2$, $\max 60 \mathrm{mg} \times 2)$.

After 1 year of treatment, there was a total regression of the left adrenal lesion, and a partial response of the right superior sulcus mass.

On the preoperative contrast-enhanced chest CT scan, the mass measured $52 \mathrm{~mm} \times 41 \mathrm{~mm}$ (Figure 1A), with some infiltration of the soft tissues in the right supraclavicular region and possibly invading the right subclavian artery (Figure 1B).

This presentation drove us to adopt a sequential surgical approach; first by exploratory neck cervicotomy for vessel reconstruction, if needed, followed by right upper robotassisted lobectomy, as a second step.

The first exploratory surgery was achieved by cervicotomy, 23/10/2018. We started by adhesiolysis, then we clipped a tumor feeding vessels arising from the right subclavian artery. Fortunately, the subclavian artery by itself, was not invaded and the procedure didn't necessitate any vascular reconstruction.

Two days later, 25/10/2018, we proceeded with a right upper robot-assisted lobectomy, associated with radical lymphadenectomy (Video 1).

The starting point was to go to the level of the upper part of the upper lobe for its dissection using monopolar scissors. The venous part is liberated by respecting the phrenic nerve. Next was the preparation and dissection the whole posterior part passing through a fibrous and extra pleural plane, which was very difficult to dissect due to the tumor and its answer to immunotherapy.

It was decided then to continue the lobectomy, even with the upper lung rest still adhesive to the level of the apex. Starting dissection is in the fissure of the pulmonary artery, the posterior part of the fissure is opened and stapled with the mechanical forceps. The lung was extremely fragile and emphysematous. Control was achieved of the pulmonary artery in the fissure and then has been resolved the A2 branch and of the A3 branch in two stages using a suitable mechanical stapler. The upper lobe bronchus was dissected after having liberated the posterior and anterior part of the bronchus. Its control and section have been provided by using a mechanical stapler. The lobectomy is terminated by separating the middle lobe from the superior lobe with a mechanical stapler, which at the same time takes the vein and the last pulmonary arterial branch. 

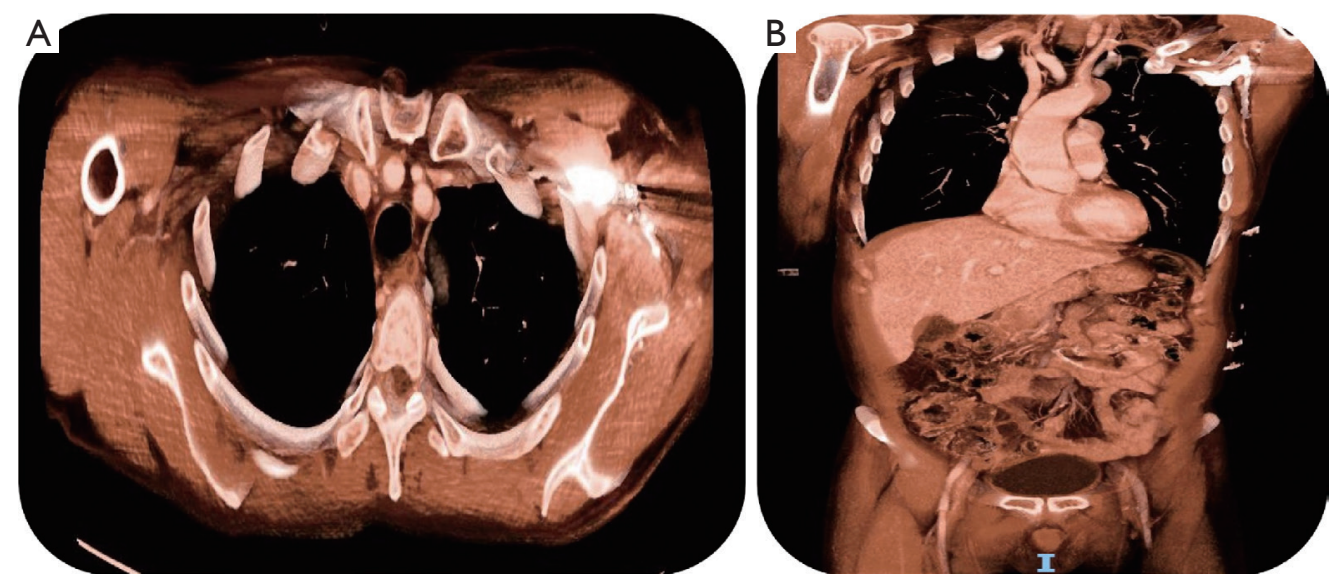

Figure 2 Post-operative computed tomography without evidence for early recurrence (A and B).

The lobectomy is completed on the hilar plane. The procedure has continued in the manner going to the level of the apical part to pursue and dissect the tumor gradually. There was a doubt about a zone of resection that sends to the immediate AnaPath investigation. We managed to lift the upper lobe passing extra-pleural in this region of the upper sulcus which was very complicate to achieve, with absence of bleeding.

The patient was discharged at day 4 after the second procedure with a total length of stay 8 days (at 2 weeks the patient was fine without major pain and return to his own activity). The only sequel was hand force diminished; unfortunately, at one month (free interval) the patient started to present a neuropathic pain which oblige to specific treatment.

Pathology: necrotic lesion of $6.5 \mathrm{~cm}$ long axis, persisting on about $1 \%$ of the examined surface of adenocarcinoma cells TTF $1+$ absence of tumor vascular embolus absence of peri-nervous tumor swelling absence of invasion of the visceral pleura by the tumor and absence of airborne tumor diffusion. The bronchial and vascular surgical limits were healthy. Lesion ypT1aN0. MSCT at 6 months after operation-no argument for early recurrence (Figure 2). Adrenal thickening left discreetly increased month later compared to the scanner before operation and it was later continued with reprise of pembrolizumab for the reason of PET positive adrenal gland. Unfortunately, at $9^{\text {th }}$ months after there was a local recurrence despite the complete resection. All procedures performed in this study were in accordance with the ethical standards of the institutional and/or national research committee(s) and with the Helsinki Declaration (as revised in 2013). Written informed consent was obtained from the patient for publication of this case report and accompanying images/video. A copy of the written consent is available for review by the editorial office of this journal.

\section{Discussion}

Several techniques have been proposed for the challenging surgical resection of superior pulmonary sulcus tumors.

The classical approach involves an extended posterolateral thoracotomy to the base of the neck so called Shaw-Paulson approach (3). However, if the posterior incision is made small, the visual field and operation technique will be compromised. With tumors invading the anterior aspects of the first rib or subclavian artery or vein, an anterior approach is helpful. Dartevelle and colleagues (4) pioneered the anterior cervicothoracic approach with an L-shaped incision paralleling the anterior border of the sternocleidomastoid muscle down the midline to the level of the second or third ribs, extending laterally to the deltopectoral groove. The medial clavicle is resected. Excellent exposure of the subclavian vessels, anterior aspects of ribs, and brachial plexus is possible through this incision. Lobectomy is performed after detachment of the chest wall.

Rush et al. (5) has described the multimodality program as the corner stone of the treatment with radio chemotherapy plus surgery. In our case we have operated the patient after immunotherapy without radiotherapy which can explain the recurrence at 9 months.

The objective of this case report is to highlight a new surgical spirit based on sequential hybrid surgery to achieve good resection in a less aggressive manner. 
A combined approach to superior sulcus tumors provides good access to both the apical and hilar sites in a less invasive manner; this allows safer dissection and better visualization, which dramatically contribute to the safety of this complex procedures.

It reduces the magnitude of surgery, by sparing the patient a full-scale thoracotomy or by optimizing the site of the thoracotomy. It may also have a significant educational role (6).

Described here is the novel use of robot-assisted thoracic surgery, rather than thoracotomy, to dissect the pulmonary vessels and perform lobectomy with an extensive lymphadenectomy after exploratory cervicotomy with adhesiolysis of the subclavian artery. We believe similar like other authors (7) that, when feasible, RATS likes VATS allows a significant reduction in surgical trauma, although a combined approach only makes sense if the surgical resection specimen is not so bulky that it cannot be easily removed from inside the chest or where are significant infiltration of other surrounding structures so R0 resection, not possible.

The minimally invasive approach seems to be of greatest utility when there is limited invasion of the apical chest wall. The future of our surgical practice will probably be based more on minimally invasive or combined approaches. The authors believe that sequential surgery could potentially represent an optimal model to solve some difficult surgical challenges.

\section{Acknowledgments}

Funding: None.

\section{Footnote}

Provenance and Peer Review: This article was commissioned by the editorial office, Fournal of Visualized Surgery for the series "Robotic Assisted Thoracic Surgery: Advanced Procedures in Lung and Mediastinum: From Postinduction TTT (immunotherapy) to Sleeve Resection, Complex Segmentectomies and Extended Thymectomy for Myasthenia Gravis". The article has undergone external peer review.

Reporting Checklist: The authors have completed the CARE reporting checklist. Available at https://jovs.amegroups. com/article/view/10.21037/jovs-20-95/rc

Conflicts of Interest: All authors have completed the
ICMJE uniform disclosure form (available at https://jovs. amegroups.com/article/view/10.21037/jovs-20-95/coif). The series "Robotic Assisted Thoracic Surgery: Advanced Procedures in Lung and Mediastinum: From Postinduction TTT (immunotherapy) to Sleeve Resection, Complex Segmentectomies and Extended Thymectomy for Myasthenia Gravis" was commissioned by the editorial office without any funding or sponsorship. JMB served as the unpaid Guest Editor of the series. The authors have no other conflicts of interest to declare.

Ethical Statement: The authors are accountable for all aspects of the work in ensuring that questions related to the accuracy or integrity of any part of the work are appropriately investigated and resolved. All procedures performed in this study were in accordance with the ethical standards of the institutional and/or national research committee(s) and with the Helsinki Declaration (as revised in 2013). Written informed consent was obtained from the patient for publication of this case report and accompanying images/video. A copy of the written consent is available for review by the editorial office of this journal.

Open Access Statement: This is an Open Access article distributed in accordance with the Creative Commons Attribution-NonCommercial-NoDerivs 4.0 International License (CC BY-NC-ND 4.0), which permits the noncommercial replication and distribution of the article with the strict proviso that no changes or edits are made and the original work is properly cited (including links to both the formal publication through the relevant DOI and the license). See: https://creativecommons.org/ licenses/by-nc-nd/4.0/.

\section{References}

1. Rusch VW. Management of Pancoast tumours. Lancet Oncol 2006;7:997-1005.

2. Truin W, Siebenga J, Belgers E, et al. The role of videoassisted thoracic surgery in the surgical treatment of superior sulcus tumors. Interact Cardiovasc Thorac Surg 2010;11:512-4.

3. Paulson DL. Technical considerations in stage III disease: the "superior sulcus" lesion. In: Delarue NC, Eschapasse $\mathrm{H}$ (eds). Lung cancer. Vol. 1 of International trends in general thoracic surgery. Philadelphia: W.B. Saunders, 1985:121-31.

4. Dartevelle PG, Chapelier AR, Macchiarini P, et al. 
Anterior transcervical-thoracic approach for radical resection of lung tumors invading the thoracic inlet. J Thorac Cardiovasc Surg 1993;105:1025-34.

5. Rusch VW, Giroux DJ, Kraut MJ, et al. Induction chemoradiation and surgical resection for superior sulcus non-small-cell lung carcinomas: long-term results of Southwest Oncology Group Trial 9416 (Intergroup Trial

doi: 10.21037/jovs-20-95

Cite this article as: Kostic M, Sarsam M, Bottet B, Baste JM. Post-immunotherapy combined operative technique with an anterior surgical approach and robot-assisted lobectomy for an anterior superior sulcus tumor-case report. J Vis Surg 2022;8:5.
0160). J Clin Oncol 2007;25:313-8.

6. Caronia FP, Ruffini E, Lo Monte AI. The use of videoassisted thoracic surgery in the management of Pancoast tumors. Interact Cardiovasc Thorac Surg 2010;11:721-6.

7. Puma F, Gili A, Cagini L, et al. Surgery for Pancoast tumors-the role of combined approaches. Curr Chall Thorac Surg 2020;2:4. 